\title{
Illusory recouplings of onsets and terminations of glide tone components
}

\author{
YOSHITAKA NAKAJIMA \\ Kyushu Institute of Design, Fukuoka, Japan \\ TAKAYUKI SASAKI \\ Miyagi Gakuin Women's College, Sendai, Japan \\ KYOKO KANAFUKA and ATSUKO MIYAMOTO \\ Kyushu Institute of Design, Fukuoka, Japan \\ and \\ GER REMIJN and GERT TEN HOOPEN \\ Leiden University, Leiden, The Netherlands
}

\begin{abstract}
We present a new auditory illusion, the gap transfer illusion, supported by phenomenological and psychophysical data. In a typical situation, an ascending frequency glide of 2,500 msec with a temporal gap of $100 \mathrm{msec}$ in the middle and a continuously descending frequency glide of $500 \mathrm{msec}$ cross each other at their central positions. These glides move at the same constant speed in logarithmic frequency in opposite directions. The temporal gap in the long glide is perceived as if it were in the short glide. The same kind of subjective transfer of a temporal gap can take place also when the stimulus pattern is reversed in time. This phenomenon suggests that onsets and terminations of glide components behave as if they were independent perceptual elements. We also find that when two long frequency glides are presented successively with a short temporal overlap, a long glide tone covering the whole duration of the pattern and a short tone around the temporal middle can be perceived. To account for these results, we propose an event construction model, in which perceptual onsets and terminations are coupled to construct auditory events and the proximity principle connects these elements.
\end{abstract}

The concept of auditory streams plays an important role when we investigate auditory organization (e.g., Bregman, 1990, 1993; Handel, 1989). An auditory stream is a temporal sequence of perceived sounds and silent intervals. A phrase in speech, a melody in music, a sequence of approaching footsteps, and the continuous sound of a waterfall are all typical auditory streams. We report a new auditory illusion that gives insight into the mechanism of auditory stream formation.

Because we found no previous literature related directly to our finding, we first would like to introduce the perceptual phenomenon itself by describing a part of our pilot study.

This research was supported by the Ministry of Education, Japan (07459017 in the fiscal years $1995-1997$ and 10610076 in the fiscal years 1998-1999) and by the Hayao Nakayama Foundation for Science, Technology, and Culture (in the fiscal year 1996). Shunsuke Tanaka gave us valuable suggestions. Stephen McAdams, Valter Ciocca, and an anonymous reviewer gave us considerable help with our manuscript. Correspondence concerning this article should be addressed to Y. Nakajima, Department of Acoustic Design, Kyushu Institute of Design, 4-9-1 Shiobaru, Minami-ku, Fukuoka 815-0032, Japan (e-mail: nakajima@kyushu-id.ac.jp).

\section{PRELIMINARY EXPERIMENT}

When an ascending and a descending glide that are reversals of each other in time are presented simultaneously, a higher and a lower component bouncing against each other are often perceived (Halpern, 1977; Tougas \& Bregman, 1985). Because the first half of the descending glide and the last half of the ascending glide are in a higher frequency region, the principle of frequency proximity seems to work to connect these parts perceptually (Bregman, 1990). The same sort of argument holds also for the first half of the ascending glide and the last half of the descending glide. Nakajima and Sasaki (1993) were interested in this phenomenon and made several stimulus patterns. Four stimulus patterns, like those in Figure 1, are important in the present context. These patterns were presented monaurally at a comfortable listening level through an earphone. Ten female music students, 20-24 years of age, listened to the patterns. They were requested to describe their percepts graphically. When the majority of these observers had the same type of perceptual impression, we regarded it as typical.

Figure 1a shows a stimulus pattern containing two glides: a long ascending frequency glide of $1,500 \mathrm{msec}$ 

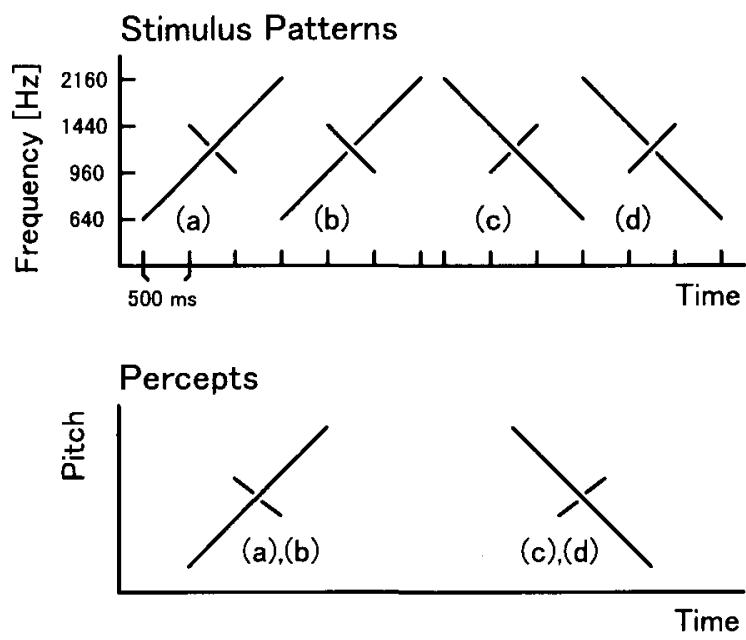

Figure 1. Stimulus patterns and typical percepts in the preliminary experiment. A long and a short glide component cross each other in each pattern. The horizontal axis represents time, whereas the vertical axis represents logarithmic frequency or pitch (see the text for details).

from 640 to $2160 \mathrm{~Hz}$ and a short descending frequency glide of $500 \mathrm{msec}$ from 1440 to $960 \mathrm{~Hz}$ with a temporal gap of $100 \mathrm{msec}$ in the middle. They had the same amplitude, and their rise and fall times were always $10 \mathrm{msec}$. The perception of this pattern was veridical. A typical percept was a long ascending component accompanied by a short descending component with a temporal gap in the middle (Table 1). It should be noted, however, that the above-mentioned bouncing perceptual components did not appear, although the frequency proximity principle was available.

Figure $1 \mathrm{~b}$ shows a new stimulus pattern, in which the temporal gap of $100 \mathrm{msec}$ was introduced into the ascending frequency glide. Surprisingly, the typical percept remained almost the same. Although the physical temporal gap was in the long ascending component, it was still perceived as being in the short descending component (Table 1). The stimulus patterns in Figures $1 \mathrm{c}$ and $1 \mathrm{~d}$ are the temporal reversals of the previous patterns. Again, the typical percept consisted of a long continuous component and a short component with a gap in the middle (Table 1).

In the stimulus patterns in Figures $1 \mathrm{~b}$ and $1 \mathrm{~d}$, an illusory transfer of the temporal gap from the longer to the shorter component took place. Nakajima and Sasaki (1993) presented the stimulus patterns or their variations to more than 50 listeners and found that the illusion was robust if the stimulus patterns were presented over headphones or a loudspeaker close enough to (approximately $70 \mathrm{~cm}$ distant from) the listener. ${ }^{1}$

Nakajima and Sasaki (1993) named the phenomenon the gap transfer illusion. One may notice that an aspect of this illusion has something in common with temporal induction (the illusion of continuity), reported briefly by Miller and Licklider (1950) and studied further by Thurlow (1957) and Elfner (1969). A theoretical discussion of temporal induction appeared in Warren $(1982,1999)$ and
Bregman (1990). In Figure 1b, for example, the long ascending component has a physical temporal gap that is long enough to be perceived, but the presence of the short descending component restores the subjective continuity of the long component. It seems as if a portion of sound energy of the shorter component around the crossing point was reallocated to the longer component, offering a typical example of temporal induction. The present phenomenon might be interpreted as an example in which the neural response to the inducer is partly used to restore the inducee (see, e.g., Warren, Bashford, Healy, \& Brubaker, 1994). If this were the case, it would be the first example in which the real portion necessary for restoration was specified clearly.

This analogy, however, has a limitation, and we do not think that the gap transfer illusion is just a variation of temporal induction. The continuity of the shorter component, which would be solid if this component were presented alone, is broken subjectively by introducing the longer component. If the principle of good continuity worked here, this would mean that the subjective continuity of one component is sacrificed for that of the other component. Why does the continuity of the longer component have priority? We will address this issue in the General Discussion section.

We report a phenomenological and a psychophysical experiment and try to find a correspondence between them. The advantages of psychophysical experiments over phenomenological experiments are that the subjects can concentrate on limited aspects of stimulus patterns and that the way to respond to the patterns is also limited, enabling us to get quantitative data efficiently. The advantages of phenomenological experiments, on the other hand, are that the observers can respond without any particular restriction and that the observers often give us qualitative information indicating the nature of the percept. Considering the advantages of both types of procedures, we conducted a phenomenological experiment and a psychophysical experiment with the same set of stimulus patterns. An additional phenomenological experiment examined whether it was possible to generalize our find-

Table 1

Number of Observers Who Reported Typical Percepts in the Preliminary Experiment

\begin{tabular}{cccc}
\hline Pattern & Typical & $\begin{array}{c}\text { Typical } \\
\text { Except for } \\
\text { Pitch Movement }\end{array}$ & Exceptional \\
\hline $\mathrm{a}$ & 9 & 0 & 1 \\
$\mathrm{~b}$ & 8 & 1 & 1 \\
$\mathrm{c}$ & 7 & 1 & 2 \\
$\mathrm{~d}$ & 6 & 0 & 4 \\
\hline
\end{tabular}

Note-The stimulus patterns and the typical percepts are shown in Figure 1. A long glide tone and two successive short tones were reported also in the eight $(1+1+2+4)$ exceptional cases, and the long glide tone was never discontinuous perceptually. This basically indicated the same type of percept as in the typical cases. These cases were considered exceptional, however, because the two short tones in the graphic descriptions were not covered fully by the duration (= the horizontal range) of the long tone. 

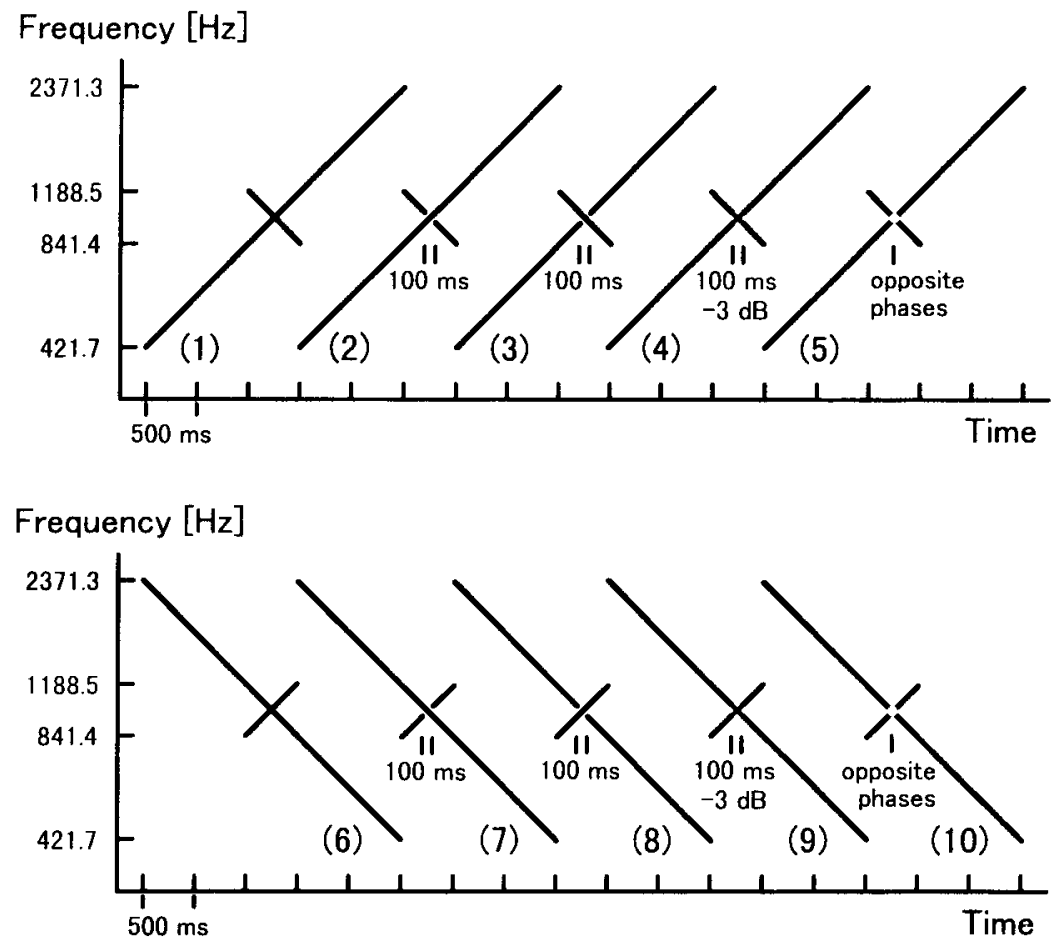

Figure 2. Stimulus patterns employed in Experiments 1 and 2. A long and a short glide component cross each other in each pattern. The horizontal axis represents time, whereas the vertical axis represents logarithmic frequency (see the text for details).

ing. These experiments demonstrated the existence and robustness of the gap transfer illusion. We then report another phenomenological experiment, which was carried out in an attempt to find another perceptual phenomenon that could introduce a more general view of the illusion. Finally, we present a simple perceptual model in the General Discussion section.

\section{EXPERIMENT 1}

This experiment was designed in order to replicate the results of the preliminary experiment. The observers were required to describe their percepts in detail, both verbally and graphically. Several phenomenological experiments of the same kind had been conducted before this experiment in the same research project (e.g., Tanaka, Nakajima, \& Sasaki, 1994), and the results of all these experiments were similar to each other. We chose the present experiment to report in this paper because it was the most systematic one.

\footnotetext{
Method

Observers. Seven observers, 4 males and 3 females, including authors Y.N., K.K., and A.M., participated. Their ages ranged between 22 and 42 years, and they had normal hearing. Six of them were students and 1 (male) was a teacher at the Kyushu Institute of Design. They had received basic training in music, and the students had received training in technical listening for acoustic engineers.

Stimuli and Apparatus. Ten stimulus patterns were employed (see Figure 2). Pattern 1 consisted of a long ascending frequency glide of 2,500 msec from 421.7 to $2371.3 \mathrm{~Hz}$ and a short descend-
}

ing frequency glide of $500 \mathrm{msec}$ from 1188.5 to $841.4 \mathrm{~Hz}$. These frequency glides moved in logarithmic frequency at a constant speed of about $1 \mathrm{oct} / \mathrm{sec}$ (exactly $0.3 / \mathrm{sec}$ on the common logarithmic scale). The glides crossed each other at $1000 \mathrm{~Hz}$ in the same phase. The rise and fall times of the long ascending frequency glide were $500 \mathrm{msec}$, and those of the short descending component were $10 \mathrm{msec}$. All the other patterns were variations of this basic pattern.

Pattern 2 had a temporal gap of $100 \mathrm{msec}$ (between 966.1 and $1035.1 \mathrm{~Hz}$ ) in the short descending frequency glide at the crossing point. The rise and fall times around the gap were $10 \mathrm{msec}$. Pattern 3 had a temporal gap of $100 \mathrm{msec}$ in the long ascending frequency glide at the crossing point. The rise and fall times around the gap were $10 \mathrm{msec}$. Pattern 4 had a temporal dip of $3.01 \mathrm{~dB}$ on both glides at the crossing point, and thus the total intensity changed in the same manner as in Patterns 2 and 3. Pattern 5 was the same as Pattern 1, except that the two glides had opposite phases at the crossing point. Patterns $6-10$ were made by reversing Patterns $1-5$ in time.

All the stimulus patterns were generated and controlled by a computer (Hewlett Packard 362) with a 16-bit digital-to-analog (D/A) converter at a sampling frequency of $20000 \mathrm{~Hz}$. An active low-pass filter at $8300 \mathrm{~Hz}$ was utilized for anti-aliasing. They were presented to the left ear of the observer in a soundproof booth at about $73 \mathrm{dBA}$ (fast-peak) through an amplifier (Sansui AU-XR) and headphones (Rion AD-02). The sound level was measured with a sound level meter and an artificial ear (Brüel \& Kjær 2209, 4152). The observer started each presentation by pressing one of the keys of a portable computer, which was connected to the computer outside the booth. There was always a silent interval of about $3 \mathrm{sec}$ between the keypressing and the onset of the pattern.

Procedure. The observer could listen to each pattern as many times as he/she wanted. The observer described the perceptual impression verbally and graphically with a pencil on a blank sheet and handed this sheet to the experimenter when he/she finished the pat- 

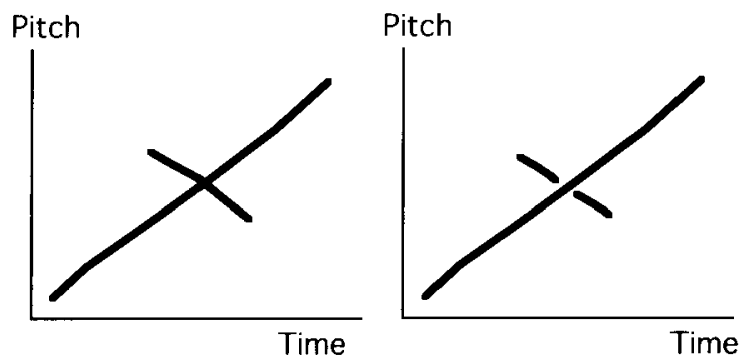

Figure 3. Typical examples of graphic descriptions by the observers in Experiment 1.

tern. If there was more than one type of percept, the observer described all the types and indicated the order of dominance. The experimenter sometimes asked a question about the observer's description when it included vague or contradictory parts. All the questions were limited to asking for a more detailed explanation, and inducing new content by way of the questioning was carefully avoided.

A training session consisting of three observations was given to each observer. These observations were carried out in the same way as those in the main sessions. Three stimulus patterns were employed for the training, so that 1 pattern was chosen from each of the following pairs: Patterns 1 and 6, Patterns 2 and 7, and Patterns 3 and 8 . At least 1 pattern was chosen from Patterns 1,2, and 3, and at least 1 from Patterns 6, 7, and 8 . After a short rest of several minutes, a warm-up of two observations was followed by the experimental trials ( 10 patterns presented in random order).

\section{Results}

Globally speaking, all the observers perceived a long component and a short component for all the stimulus patterns. The short component was located around the temporal middle of the long component. Typical examples of graphic descriptions are shown in Figure 3.

Figure 3 (right panel) shows how an observer perceived Pattern 3 . Although the long frequency glide had a temporal gap in the middle, the gap was perceived as if it had been in the short glide, and the long component was perceived as continuous. This was what we call the gap transfer illusion.

The observers' reports included sufficient information to classify the continuity-discontinuity of each perceptual component into three categories, as follows: (1) completely continuous, (2) weak evidence of discontinuity (as a dip or a crack), and (3) completely discontinuous. The frequencies of these categories are indicated in Table 2. We checked whether the longer or the shorter perceptual component was more discontinuous and conducted a sign test (two-tailed) between them for each stimulus pattern. The results are shown in Table 2. The sign test for each stimulus pattern was based on seven comparisons for the 7 observers. When both perceptual components belonged to Category 1 or to Category 3 , the comparison was regarded as a tie and was not included in the sign test. When both components belonged to Category 2 , we checked the graphical and the verbal report closely in order to determine which perceptual component was more discontinuous.
All the observers showed the same type of perception for Patterns 2, 3, 7, and 8. The longer perceptual component was always completely continuous, and the shorter component was always completely discontinuous. There were no exceptional cases.

\section{Discussion}

The results corresponded to those of the preliminary experiment. Although we conducted no statistical test in the preliminary experiment, the same type of results would appear for the data in Table 1, because the shorter perceptual component was always more discontinuous than the longer one. The results for Patterns 3 and 8 in the present experiment supported the existence of the gap transfer illusion.

Pattern 1 and Pattern 5 each consisted of two continuous frequency glides whose gliding speeds in logarithmic frequency were constant and equal to each other. All the observers reported a long component and a short component (as is indicated in Figure 3), and these perceptual components seemed to correspond to the two physical glides. Although it is well known that bouncing percepts often appear in this kind of stimulus pattern, McPherson, Ciocca, and Bregman (1994) found that crossing percepts can be dominant when two crossing glides had different gliding rates (= speeds). It would be productive in the future to clarify the physical conditions in which we can obtain crossing percepts.

One observer reported a third perceptual component, which sounded far lower in pitch than the other components at any time. This component started and ended simultaneously with the short component, which crossed the long component. The temporal gap in the abovementioned short component was shared by this component, which descended before and ascended after the gap. This was probably the percept of one of the combination tones $\left(f_{2}-f_{1}\right)$ of the two physical glides. Its symmetrical movement (descending and ascending) in time

Table 2

Discontinuities of Perceptual Components in Experiment 1

\begin{tabular}{|c|c|c|c|c|c|c|c|}
\hline \multirow[b]{3}{*}{ Pattern } & \multicolumn{6}{|c|}{ Discontinuity } & \multirow[b]{3}{*}{ Sign Test } \\
\hline & \multicolumn{3}{|c|}{$\begin{array}{c}\text { Long } \\
\text { component }\end{array}$} & \multicolumn{3}{|c|}{$\begin{array}{c}\text { Short } \\
\text { component }\end{array}$} & \\
\hline & 1 & 2 & 3 & 1 & 2 & 3 & \\
\hline 1 & 5 & 2 & 0 & 5 & 0 & 2 & \\
\hline 2 & 7 & 0 & 0 & 0 & 0 & 7 & $<$ \\
\hline 3 & 7 & 0 & 0 & 0 & 0 & 7 & $<$ \\
\hline 4 & 7 & 0 & 0 & 3 & 1 & 3 & \\
\hline 5 & 5 & 2 & 0 & 1 & 3 & 3 & $<$ \\
\hline 6 & 7 & 0 & 0 & 4 & 1 & 2 & \\
\hline 7 & 7 & 0 & 0 & 0 & 0 & 7 & $<$ \\
\hline 8 & 7 & 0 & 0 & 0 & 0 & 7 & $<$ \\
\hline 9 & 7 & 0 & 0 & 1 & 3 & 3 & $<$ \\
\hline 10 & 5 & 2 & 0 & 1 & 2 & 4 & $<$ \\
\hline
\end{tabular}

Note-The discontinuity of each component was classified into the following three categories: (1) continuous, (2) partially discontinuous, and (3) discontinuous. The frequency of each category is indicated for each perceptual component. $<$, the short component is significantly more discontinuous than the long component at the $5 \%$ level. 


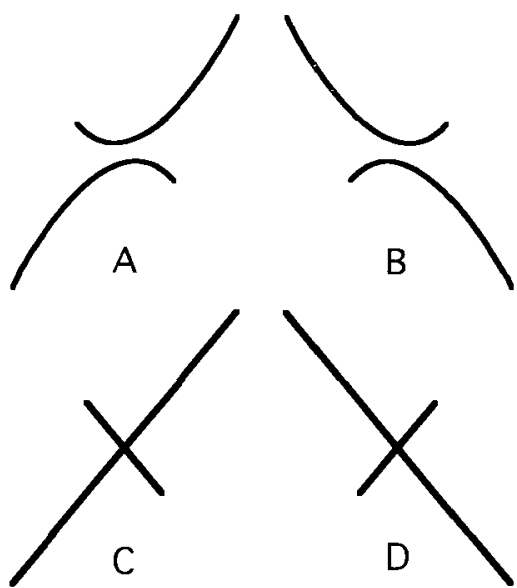

Figure 4. Four perceptual categories given to the observers as possible alternatives in Experiment 2.

corresponded to the symmetrical movement of the two glides in the overlapping parts. This was the only case in this experiment and in the preliminary experiment where any combination tones were identified in the percepts. This observer proved the robustness of the gap transfer illusion in a sense, because the illusion took place, in Patterns 3 and 8 , even when his observation was keen enough to detect the combination tone clearly.

On the other hand, this exceptional observer indicated a problem. The two short tones reported by the other observers for Patterns 3 and 8 may have been combination tones (typically, $f_{2}-f_{1}$ or $2 \times f_{1}-f_{2}$ ). In this case, however, the pitch movements of the combination tones should be in opposite directions before and after the temporal gap, because the stimulus patterns were symmetrical in time for these parts. This was indeed the case for the third perceptual component reported by the above-mentioned exceptional observer. The other observers, however, did not report a third perceptual component, and they did not indicate that the two short tones moved in opposite directions. Rather, for Pattern 3, all of them reported that descending pitch movement appeared, or at least dominated, in both short tones. For Pattern 8, 5 of them reported that both short tones ascended. One observer reported that the first short tone ascended in the last portion and that the second short tone had a constant pitch. Thus, it is unlikely that these observers reported any combination tones. The pitch movement they reported supports the idea that the temporal gap in the long glide component transferred to the short glide component perceptually.

Another point is to be noticed in the present context. If the two short tones in the percept of the gap transfer illusion had been combination tones, most observers must have completely missed the short continuous glide as an independent component. This is also quite unlikely.

\section{EXPERIMENT 2}

It seemed important to examine whether we could derive the same conclusion also from quantitative data. This experiment was conducted to prove the existence of the gap transfer illusion in a psychophysical paradigm.

\section{Method}

Observers. Twenty observers, 11 males and 9 females, including authors Y.N. and K.K., participated. Their ages ranged between 18 and 42 years, and they had normal hearing. Nineteen of them were students, and 1 (male) was a teacher at the Kyushu Institute of Design. They had received basic training in music, and the students had received training in technical listening for acoustic engineers.

Stimuli and Apparatus. The same stimulus patterns as those in Experiment 1 were generated and controlled with the same apparatus.

Procedure. The observer first had to judge whether the pattern as a whole was ascending or descending and whether the percept was bouncing or crossing. He/she chose one or more among the following categories (Figure 4).

(A) Two tones bounce against each other. Because the ascending parts are longer than the descending parts in both tones, the pattern ascends as a whole.

(B) Two tones bounce against each other. Because the descending parts are longer than the ascending parts in both tones, the pattern descends as a whole.

(C) A long ascending tone is accompanied by a short tone around its temporal middle. (These tones typically cross each other.)

(D) A long descending tone is accompanied by a short tone around its temporal middle. (These tones typically cross each other.)

(E) Other percepts.

Categories A-D were indicated in random order on each response sheet with graphic illustrations. The last, Category E, was added to allow the observers to report any other percepts.

The observer had to judge the continuity (or discontinuity) of each perceptual component. The upper and the lower component in Categories $\mathrm{A}$ and $\mathrm{B}$ and the longer and the shorter component in Categories $\mathrm{C}$ and $\mathrm{D}$ were provided with 4-point scales of continuitydiscontinuity. The number of the scale points had been determined in a pilot study.

A response sheet was prepared for each stimulus pattern. The observer could listen to the pattern at any time in the trial by pressing a key (of the portable computer), as in Experiment 1 . He/she could select more than one category, giving rankings of dominance to all the chosen categories. If a percept was not classified into Categories A-D, the observer described it verbally and graphically.

The 10 stimulus patterns appeared in random order, making 10 trials in each session. Three sessions were given to each observer, and the last two sessions were analyzed as data. Two warm-up trials were given before the second and the third session and after any rest.

\section{Results}

When two or more categories were chosen, only the most dominant one was analyzed. Fifteen of the $20 \mathrm{ob}-$ servers judged that the patterns in all the analyzed trials consisted of one long component and one short component sharing the temporal middle (Categories $\mathrm{C}$ and $\mathrm{D}$ ), indicating crossing percepts or similar types of percepts. Three other observers made judgments of this kind in at least 17 out of the 20 trials. It seems safe to regard such percepts as typical and to regard other types (e.g., bouncing percepts) as exceptional. If the judgments of these 18 observers $(=15+3)$ are combined, omitting 2 observers, we get 36 or 35 typical judgments in total for each pattern.

The other 2 observers were not included in the subsequent analyses. One of them made typical judgments only in 13 trials, and the other only in 10 trials. This means that 
Table 3

Discontinuities of Perceptual Components in Crossing Percepts in Experiment 2

\begin{tabular}{|c|c|c|c|c|c|c|c|c|c|}
\hline \multirow[b]{3}{*}{ Pattern } & \multicolumn{8}{|c|}{ Discontinuity } & \multirow[b]{3}{*}{ Sign Tes } \\
\hline & \multicolumn{4}{|c|}{$\begin{array}{c}\text { Long } \\
\text { component }\end{array}$} & \multicolumn{4}{|c|}{$\begin{array}{c}\text { Short } \\
\text { component }\end{array}$} & \\
\hline & 0 & 1 & 2 & 3 & 0 & 1 & 2 & 3 & \\
\hline 1 & 35 & 1 & 0 & 0 & 15 & 8 & 7 & 6 & $<<$ \\
\hline 2 & 36 & 0 & 0 & 0 & 0 & 3 & 5 & 28 & $<<$ \\
\hline 3 & 33 & 1 & 0 & 1 & 1 & 5 & 3 & 26 & $<<$ \\
\hline 4 & 35 & 0 & 0 & 0 & 13 & 8 & 6 & 8 & $<<$ \\
\hline 5 & 27 & 7 & 1 & 0 & 8 & 13 & 6 & 8 & $<<$ \\
\hline 6 & 33 & 2 & 0 & 0 & 15 & 9 & 3 & 8 & $<<$ \\
\hline 7 & 34 & 2 & 0 & 0 & 1 & 1 & 8 & 26 & $<<$ \\
\hline 8 & 34 & 1 & 0 & 0 & 1 & 4 & 5 & 25 & $<<$ \\
\hline 9 & 33 & 3 & 0 & 0 & 8 & 11 & 8 & 9 & $<<$ \\
\hline 10 & 31 & 2 & 2 & 0 & 6 & 14 & 9 & 6 & $<<$ \\
\hline
\end{tabular}

Note-The frequency of each response category is indicated for each perceptual component. Each of the 18 observers analyzed here responded twice for each stimulus pattern. However, the total frequency for each component could be below $36(=2 \times 18)$, because some trials were omitted from the analysis when the percepts were not crossing. $<<$, the short component is significantly more discontinuous than the long component at the $1 \%$ level.

they made exceptional judgments in 17 trials in total. Their percepts were bouncing in most of these cases.

The results are summarized in Table 3 . The long perceptual component was almost perfectly continuous in all the patterns except for Patterns 5 and 10, where more intermediate responses appeared than in the other patterns. We conducted sign tests (two-tailed) for the judged continuity-discontinuity between the long and the short component. The 36 trials by the 18 observers were considered as independent comparisons of continuitydiscontinuity between the two perceptual components. Trials in which exceptional percepts appeared were omitted. The shorter component was significantly more discontinuous than the longer component at the $1 \%$ level in all the patterns.

\section{Discussion}

It may seem strange at first that discontinuity appeared in Patterns 1, 5, 6, and 10, where there seem to have been no physical gaps or dips. However, the patterns did have temporal gaps of considerable durations in their temporal envelopes, which may have been neglected in previous studies in which crossing frequency glides were employed. Figure 5 shows the acoustic beats between the two crossing frequency glides in Patterns 1 and 5 (or in Patterns 6 and 10). The beat frequency was lower near the crossing point, where these glides had similar frequencies. Pattern 1 had two temporal gaps of about $14 \mathrm{msec}$ around the crossing point if we assume, for convenience, that the boundaries of a gap were located at the points at which the sound pressure level was $3 \mathrm{~dB}$ below the peak level. Pattern 5 had a gap of about $38 \mathrm{msec}$ exactly at the crossing point. Although these temporal gaps might have affected the perception of these patterns, as was indicated in a previous study (Tanaka et al., 1994), it was impossible to remove them completely as long as we employed crossing glides.

The temporal gap of about $38 \mathrm{msec}$ made by the acoustic beats between the two frequency glides in Patterns 5 and 10 was perceived basically as a discontinuity of the shorter perceptual component. Temporal gaps at or around the crossing point could belong both to the longer and to the shorter glide physically, but the perceptual discontinuity of the shorter component seemed far clearer than that of the longer component. The perceptual components in Patterns 5 and 10 tended to be more discontinuous than those in Patterns 1 and 6, where the temporal gaps made by the beats were shorter. Thus, the perceptual system seemed to respond to and differentiate the physical discontinuities included in these patterns. However, the discontinuities tended to be allocated to the shorter component perceptually, regardless of their physical allocation. This tendency was robust.

The tendency of the shorter perceptual component to be perceived as more discontinuous than the longer perceptual component in Patterns 3 and 8 may seem to be sufficient evidence of the gap transfer illusion. If transfer occurred, however, the temporal gap in the longer glide increased the perceptual discontinuity of the shorter perceptual component in these patterns. We compared the discontinuity ratings for the shorter perceptual components among different patterns by sign tests (two-tailed), as we did between the longer and the shorter component in each pattern. The ratings for the longer perceptual components were also analyzed in the same manner, but the latter analysis did not give much information. The longer perceptual component was more discontinuous in Pattern 5 than in the other patterns, which could be attributed to the beats between the two frequency glides.

The comparisons among the shorter components were more interesting. We can summarize the results as follows. (1) The shorter perceptual component was the least discontinuous in Patterns 1 and 6, where the two frequency glides crossed each other when they had identical phases, minimizing the effect of the acoustic beats. The shorter perceptual components were significantly more continuous in these patterns than in Patterns 5 and 10 , respectively, where the phase relationship was changed. (2) The shorter perceptual component was the most discontinuous in Patterns 2, 3, 7, and 8, where one of the frequency glides had a temporal gap of $100 \mathrm{msec}$. The shorter perceptual components in these patterns were significantly more discontinuous than those in any other patterns, and there was no significant difference among these patterns.

Remarkably, the shorter perceptual components were more discontinuous in Patterns 3 and 8 than in Patterns 1 and 5 , despite the fact that the shorter frequency glides were clearly continuous in Patterns 3 and 8, where acoustic beats did not appear around the crossing point. This may seem strange at first. In Patterns 3 and 8, however, the longer frequency glide included a temporal gap, which was as long as the gap in the shorter frequency glide in 
(1)

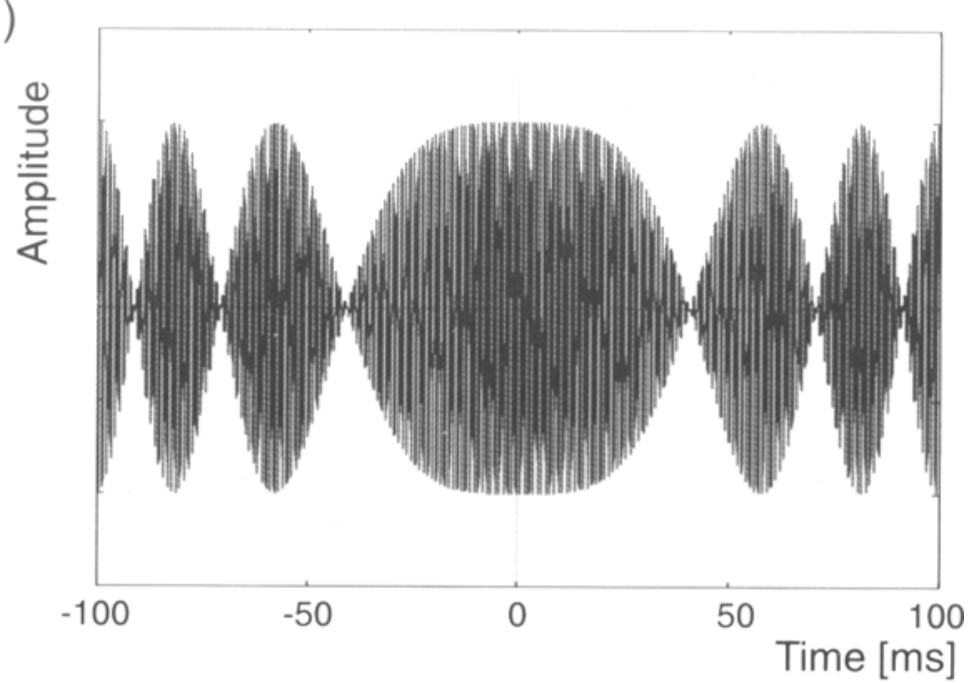

(5)

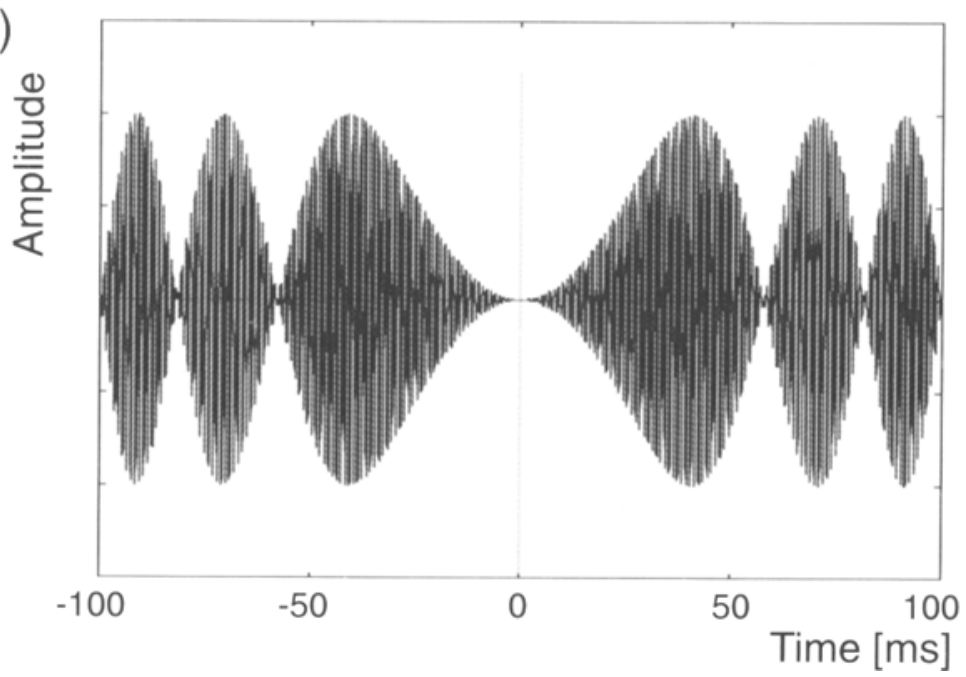

Figure 5. Waveforms around the crossing point in Pattern 1 (or Pattern 6) and Pattern 5 (or Pattern 10) in Experiments 1 and 2. Pattern 1 and Pattern 5 had different temporal envelopes around the crossing point, because the two glides had same phases in Pattern 1 and opposite phases in Pattern 5 at the crossing point.

Patterns 2 and 7. This is the only possible reason that the shorter perceptual component was clearly discontinuous in Patterns 3 and 8 . The whole picture suggests that the temporal gap of $100 \mathrm{msec}$ transferred subjectively from the longer to the shorter component. The results of the present psychophysical experiment correspond to the phenomenological data obtained in Experiment 1, and the gap transfer illusion appeared again.

It is known that our central nervous system has neurons responding to onsets or terminations (offsets) of continuous stimuli (e.g., Buser \& Imbert, 1992). Onsets of ascending or descending glides can even form an auditory stream, as was described by Bregman (1990). The present illusion seems to show that onsets and terminations of tones sometimes behave as if they were independent. In Pattern 3, for example, the onset of the descending glide and the termination of the first half of the ascending glide are very close to each other in time and (logarithmic) frequency, and the proximity principle may work to couple them. If we assume that they are coupled to make an auditory event, the appearance of the first short tone just before the gap in the typical percept is explained. The appearance of the second short tone in the percept can be explained in the same way.

\section{EXPERIMENT 3}

The temporal envelope of Patterns 3 and 8 in Experiments 1 and 2 was very clear and almost the same as that of Patterns 2 and 7 . That is, there was no level fluctuation except for the rises and the falls of the glides. Although this did not at all explain the fact that the temporal gap 
Table 4

Discontinuities of Perceptual Components in Experiment 3

\begin{tabular}{|c|c|c|c|c|c|c|c|}
\hline \multirow[b]{3}{*}{ Pattern } & \multicolumn{6}{|c|}{ Discontinuity } & \multirow[b]{3}{*}{ Sign Test } \\
\hline & \multicolumn{3}{|c|}{$\begin{array}{l}\text { Long } \\
\text { component }\end{array}$} & \multicolumn{3}{|c|}{$\begin{array}{c}\text { Short } \\
\text { component }\end{array}$} & \\
\hline & 1 & 2 & 3 & 1 & 2 & 3 & \\
\hline 1 & 7 & 0 & 0 & 4 & 2 & 1 & \\
\hline 2 & 7 & 0 & 0 & 0 & 2 & 5 & $<$ \\
\hline 3 & 6 & 1 & 0 & 1 & 1 & 5 & $<$ \\
\hline $2^{\prime}$ & 7 & 0 & 0 & 0 & 0 & 7 & $<$ \\
\hline $3^{\prime}$ & 5 & 2 & 0 & 0 & 2 & 5 & $<$ \\
\hline 6 & 6 & 0 & 0 & 3 & 1 & 2 & \\
\hline 7 & 7 & 0 & 0 & 1 & 2 & 4 & $<$ \\
\hline 8 & 7 & 0 & 0 & 1 & 1 & 5 & $<$ \\
\hline $7^{\prime}$ & 7 & 0 & 0 & 0 & 0 & 7 & $<$ \\
\hline $8^{\prime}$ & 6 & 0 & 0 & 0 & 1 & 5 & $<$ \\
\hline
\end{tabular}

Note--The discontinuity of each component was classified into the following three categories: (1) continuous, (2) partially discontinuous, and (3) discontinuous. The frequency of each category is indicated for each perceptual component. $<$, the short component is significantly more discontinuous than the long component at the $5 \%$ level.

was always perceived in the shorter component, it seemed necessary to check whether such a clear-cut temporal envelope was a requisite of the gap transfer illusion. We replaced the single-component glides with 0.05 -octave ascending or descending noises, whose fluctuating temporal envelopes would obscure the envelopes of the whole stimulus patterns.

\section{Method}

Observers. Seven observers, 5 males and 2 females, participated. Their ages ranged between 22 and 25 years, and they had normal hearing. All of them were students at the Kyushu Institute of Design. They had received basic training in music, and 6 of them had received training in technical listening for acoustic engineers.

Stimuli and Apparatus. Ten stimulus patterns were employed. Patterns 1, 2, 3, 6, 7, and 8 were the same as those in Experiments 1 and 2 , except that the single-component frequency glides were replaced with 0.05 -octave ascending or descending noises. Patterns $2^{\prime}, 3^{\prime}, 7^{\prime}$, and $8^{\prime}$ were variations of Patterns $2,3,7$, and 8 , respectively. The temporal gaps of these patterns were $250 \mathrm{msec}$. Even when the two noise components were the closest to each other, the difference in their center frequencies $(173 \mathrm{~Hz})$ was slightly larger than the corresponding critical bandwidth (160 Hz; Zwicker \& Fastl, 1999). Each sweeping noise consisted of 30 randomly chosen ascending or descending frequency glides of the same gliding speed (about $1 \mathrm{oct} / \mathrm{sec}$ ) in a range of 0.05 octave. A different set of frequency glides was generated for each observer. All the stimulus patterns were generated by a portable computer (Dell Latitude LM M166ST) with a 16-bit D/A converter at a sampling frequency of $22050 \mathrm{~Hz}$. No anti-aliasing filter was utilized, because virtually all the expected aliasing components for the present stimuli were above the highest audible frequency. They were presented to the left ear of the observer in a soundproof room at about $75 \mathrm{dBA}$ (fast-peak) through headphones (Sony MDR-CD770). The sound level was measured with a sound level meter and an artificial ear (Brüel \& Kjær 2209, 4153). The observer started each presentation by pressing the space bar of the portable computer. There was always a silent interval of about $2 \mathrm{sec}$ between the keypressing and the onset of the pattern.

Procedure. The observer first listened to all the stimulus patterns once each in random order without performing any task. Then he/she received a training trial with one randomly chosen pattern. The rest of the procedure was the same as that in Experiment 1.

\section{Results}

The results were very similar to those in Experiment 1 for Patterns 1, 2, 3, 6, 7, and 8. The results for Patterns $2^{\prime}, 3^{\prime}, 7^{\prime}$, and $8^{\prime}$ were similar to those for Patterns $2,3,7$, and 8 , respectively. The data were processed in the same way as those in Experiment 1 and are summarized in Table 4 (exceptional percepts were omitted from this analysis).

\section{Discussion}

The temporal envelopes of the stimulus patterns in the present experiment were drastically different from those in Experiments 1 and 2. In general, it was difficult to visually identify the physical temporal gap in the waveform. Nevertheless, the gap transfer illusion took place clearly. The clear-cut temporal envelopes of the original stimulus patterns do not seem essential in causing the illusion.

The illusion appeared also in Patterns $3^{\prime}$ and $8^{\prime}$, where the frequency distance between both sweeping noises was always larger than a critical bandwidth. Although the long sweeping noise was interrupted by the gap, the short sweeping noise continued to descend or ascend for more than a critical bandwidth. The gap seemed to give enough time for the peripheral system to detect the frequency movement of the short noise (Tsumura, Nakajima, \& Teranishi, 1991). Nevertheless, this portion of the short noise could be utilized by the perceptual system to fill the gap of the long noise, where the frequency movement was in the opposite direction. The results suggest that a higher level mechanism that organizes the whole auditory pattern plays an important role in this illusion.

\section{EXPERIMENT 4}

This experiment had the purpose of testing the notion that an onset and a termination from different physical sounds can be combined to give rise to a single percept. This way of thinking appeared in the discussion for Experiment 2 . The patterns that were used consisted of two parallel glides overlapping each other for a short period. These glides were separated by various intervals in logarithmic frequency (as in Figure 6). For example, the first frequency glide moved from 367.2 to $965.7 \mathrm{~Hz}$, taking $1,400 \mathrm{msec}$, and the second frequency glide moved from 1035.5 to $2723.7 \mathrm{~Hz}$, also taking $1,400 \mathrm{msec}$. These ascending glides were at a constant speed in logarithmic frequency and overlapped each other for $200 \mathrm{msec}$, keeping a distance of 0.3 octave. The onset of the second component and the termination of the first component were close to each other, making this stimulus pattern comparable to Patterns 3 and 8 in Experiments 1,2, and 3. If the proximity principle can cause an illusory recoupling of the onset and the termination, this pattern should generate an illusory short tone. That is, a typically predicted percept for the whole pattern should be that of a long ascending tone, with a short tone around the temporal middle. 


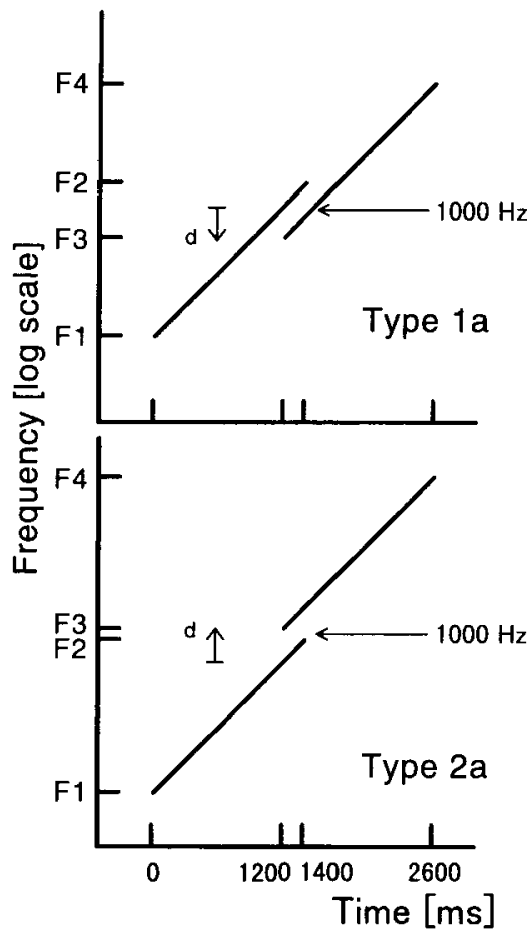

Figure 6. Stimulus patterns employed in Experiment 4. The horizontal axis represents time, whereas the vertical axis represents frequency on a logarithmic scale. The frequency distance between the two glides $(d)$ was $-0.1,-0.3,-1.3$, or -2.3 oct in Type $1 \mathrm{a}$ and $0.1,0.3,1.3$, or 2.3 oct in Type $2 \mathrm{a}$. When the frequency distance is negative, the second glide is lower than the first one in the overlapping portion. Types $1 \mathrm{~d}$ and $2 \mathrm{~d}$ are the temporal reversals of 1 and $2 a$.

We conducted a phenomenological experiment in order to examine this prediction.

\section{Method}

Observers. Three males, 22-39 years of age, participated. They had normal hearing. They were a student at the Kyushu Institute of Design, a student at Leiden University (author G.R.), and a teacher at the Kyushu Institute of Design (author Y.N.). All of them had received training in music, and 1 of them had received training in technical listening.

Stimuli and Apparatus. Each stimulus pattern consisted of two pure frequency glides of $1,400 \mathrm{msec}$. The glides ascended or descended on the logarithmic frequency scale at a speed of about $1 \mathrm{oct} / \mathrm{sec}$ (exactly $0.3 / \mathrm{sec}$ on the common logarithmic scale). The second glide started $200 \mathrm{msec}$ before the first glide ended. The first glide had a rise time of $500 \mathrm{msec}$ and a fall time of $4 \mathrm{msec}$, and the second glide had a rise time of $4 \mathrm{msec}$ and a fall time of $500 \mathrm{msec}$.

Four stimulus types were designed (Figure 6). The stimulus patterns of Type la consisted of two ascending glides, and the first glide was higher in frequency than the second glide when these glides overlapped. The stimulus patterns of Type $2 \mathrm{a}$ also consisted of two ascending glides, and the first glide was lower in frequency when these glides overlapped. The stimulus patterns of Types Id and $2 \mathrm{~d}$ were formed by reversing the stimulus patterns of Types $1 \mathrm{a}$ and $2 \mathrm{a}$, respectively, in time, and we thus got patterns consisting of descending glides. The two frequency glides in each stimulus pattern overlapped each other for $200 \mathrm{msec}$ and were separated by $0.1,0.3$, 1.3 , and 2.3 octaves. The four types and the four steps of frequency separation resulted in 16 stimulus patterns, indicated in Table 5.
The stimulus patterns were generated by a computer system (Teac PS 9000-216) with a 12-bit D/A converter at a sampling frequency of $20000 \mathrm{~Hz}$. An active low-pass filter of $7000 \mathrm{~Hz}$ was utilized for anti-aliasing. They were recorded onto a digital audio tape with a DAT deck (Sony DTC-M 100) repeatedly, with a 5-sec silence after each repetition. A DAT deck (Sony $500 \mathrm{ES}$ ), an amplifier (Sansui AU $607 \mathrm{KX}$ ), and headphones (Rion AD-02) were used to present the patterns. The sound level was measured with a sound level meter and an artificial ear (Brüel \& Kjær 2209, 4152).

Procedure. The recorded stimulus patterns were played and presented monaurally to the observer in a soundproof booth at about $67 \mathrm{dBA}$ (fast-peak). The observer reported the percept of each pattern verbally. He started and stopped the DAT player himself whenever he wanted and listened to each pattern as many times as he wanted. The experimenter recorded the observer's report and asked questions when the report included vague or contradictory parts. All the questions were limited to asking for a more detailed explanation, and inducing new content was avoided.

Training on 1 randomly chosen pattern was followed by real observations of the 16 patterns in random order.

\section{Results}

All the percepts reported by the observers could be classified into the following three modes, as is indicated in Figure 7: In Mode 1, one ascending or descending pure component was accompanied by a short nonpure component in its temporal middle; in Mode 2, two long ascending or descending components appeared successively overlapping each other often, and they were accompanied by a short (pure or nonpure) component between them; in Mode 3, two long ascending or descending components appeared successively, overlapping each other often, without any other components. The observers' perceptual impressions of each stimulus pattern were classified as is indicated in Table 5. The mode numbers indicated above were used in this table.

The general tendency was that Mode 1 appeared when the frequency separation was small and Mode 3 appeared when the separation was large. Mode 2 seemed to be intermediate between Modes 1 and 3.

\section{Discussion}

This experiment tested the idea that an onset and a termination close to each other can be recoupled illusorily. This idea was confirmed by the existence of the short middle components in Modes 1 and 2 for all the present observers. The short perceptual component in Modes 1 and 2 does not have any corresponding physical component. This perceptual component is interpreted as resulting from a perceptual recoupling of the onset of the second glide and the termination of the first glide.

It is surprising that the perceptual recoupling in Mode 2 took place even when a frequency jump of $1.3 \mathrm{oc}-$ taves was required. This strongly suggests that this kind of illusory recoupling of an onset and a termination cannot be attributed simply to the activity of the peripheral auditory system associated with critical bands or auditory filters.

One may suspect, as in Experiment 1, that the observers may have heard a combination tone of the two glides as the illusory short tone. This idea, however, does 
Table 5

Stimulus Conditions and Results of Experiment 4

\begin{tabular}{|c|c|c|c|c|c|c|c|c|}
\hline \multirow[b]{2}{*}{ Type } & \multirow{2}{*}{$\begin{array}{l}\text { Distance Between } \\
\text { Components (oct) }\end{array}$} & \multirow[b]{2}{*}{$F 1$} & \multirow[b]{2}{*}{$F 2$} & \multirow[b]{2}{*}{$F 3$} & \multirow[b]{2}{*}{$F 4$} & \multicolumn{3}{|c|}{ Perceptual Mode } \\
\hline & & & & & & Observer Y.N. & Observer S.T. & Observer G.R \\
\hline \multirow[t]{4}{*}{ la } & 0.1 & 421.7 & 1109.3 & 901.5 & 2371.1 & 1 & 1 & 1 \\
\hline & 0.3 & 452.0 & 1188.9 & 841.1 & 2212.3 & $1(2)$ & 2 & $1(2)$ \\
\hline & 1.3 & 639.2 & 1681.4 & 594.7 & 1564.3 & 2 & 3 & 2 \\
\hline & 2.3 & 904.0 & 2377.9 & 420.5 & 1106.2 & $3(2)$ & 3 & $3(2)$ \\
\hline \multirow[t]{4}{*}{$2 \mathrm{a}$} & 0.1 & 393.5 & 1035.0 & 966.2 & 2541.3 & 1 & 1 & 1 \\
\hline & 0.3 & 367.2 & 965.7 & 1035.5 & 2723.7 & 1 & $1(2)$ & 1 \\
\hline & 1.3 & 259.6 & 682.9 & 1464.4 & 3851.9 & 2 & 3 & $2(3)$ \\
\hline & 2.3 & 183.6 & 482.9 & 2071.0 & 5447.3 & $3(2)$ & 3 & $3(2)$ \\
\hline \multirow[t]{4}{*}{$1 d$} & 0.1 & 2371.1 & 901.5 & 1109.3 & 421.7 & $1(2)$ & 1 & 1 \\
\hline & 0.3 & 2212.3 & 841.1 & 1188.9 & 452.0 & 2 & 2 & $2(1)$ \\
\hline & 1.3 & 1564.3 & 594.7 & 1681.4 & 639.2 & 2 & 3 & 2 \\
\hline & 2.3 & 1106.2 & 420.5 & 2377.9 & 904.0 & $3(2)$ & 3 & $3(2)$ \\
\hline \multirow[t]{4}{*}{$2 d$} & 0.1 & 2541.3 & 966.2 & 1035.0 & 393.5 & $1(2)$ & 1 & 1 \\
\hline & 0.3 & 2723.7 & 1035.5 & 965.7 & 367.2 & 2 & $1(2)$ & $1(2)$ \\
\hline & 1.3 & 3851.9 & 1464.4 & 682.9 & 259.6 & $3(2)$ & 3 & 2 \\
\hline & 2.3 & 5447.3 & 2071.0 & 482.9 & 183.6 & $3(2)$ & 3 & $3(2)$ \\
\hline
\end{tabular}

Note-The conditions correspond to those in the caption of Figure 6, and the perceptual impressions correspond to those in Figure 7 . The modes in parentheses indicate secondary percepts that appeared just temporarily or with particular effort on the part of the observers. Frequencies are given in hertz.

not seem to hold for the patterns in which the frequency distance was 1.3 octaves. It seems unlikely for a clear combination tone to take place in such conditions, but we need further empirical data on this issue (Sasaki \& Nakajima, 1996).

\section{GENERAL DISCUSSION}

It might be helpful to consider possible explanations for the gap transfer illusion, as in Patterns 3 and 8 in Experiment 1 , by assuming peripheral auditory mechanisms. The first possible explanation is that the observer hears combination tones or acoustic beats caused by the ascending and the descending glides as short tones. Since the glides move symmetrically in time when they overlap, the expected combination tones or acoustic beats are also symmetric in time. However, the percepts of the two short tones were asymmetric in time in their pitch movement. The second possible explanation is that the temporal envelope might give a perceptual clue for the two short tones. Here again, that the pitch of the two short tones moves asymmetrically in time cannot be explained. Any explanation based on direct peripheral interaction or integration does not seem to explain the asymmetry in time in the percepts of the short tones. Furthermore, the results of Experiment 3 show that the illusion still appears even when the acoustic beats and the temporal envelope are obscured by utilizing sweeping noises.

Another possible explanation is that because the two glides are close enough in frequency to fuse around the gap, the temporal gap in the long glide (Patterns 3 and 8 in Experiments 1, 2, and 3) and that in the short glide (Patterns 2 and 7) cannot be differentiated. One may also argue, in a similar manner, that the illusion just reflects the fact that the peripheral excitation patterns in Patterns 3 and 8 are very similar to those in Patterns 2 and 7 . However, the two sweeping noises in Patterns $3^{\prime}$ and $8^{\prime}$ in Ex- periment 3 always kept a distance larger than a critical bandwidth, and it is difficult to apply the present explanation in these cases. Furthermore, an explanation of this kind offers no particular prediction about the allocation of the perceived gap. Any explanation of the gap transfer illusion is insufficient unless it can be predicted that the gap is always perceived in the shorter component. In fact, any peripheral explanation would fail in describing why the gap is always transferred from the longer to the shorter component and not vice versa, and it seems necessary to introduce a higher auditory mechanism. This does not at all mean that it is unnecessary to examine the roles of peripheral mechanisms. However, it seems important at this stage to offer a rough sketch of a simple perceptual model that covers the whole set of data. Thus, we concentrate on a model that utilizes a Gestalt principlethat is, the principle of proximity.

The present set of data introduced a hypothesis that onsets and terminations of sounds are detected independently and that they are organized perceptually to form auditory events obeying the principle of proximity. Although this idea, which we call the event construction model (Nakajima \& Sasaki, 1996), might be somewhat too simple to apply to our everyday auditory experiences, it seems

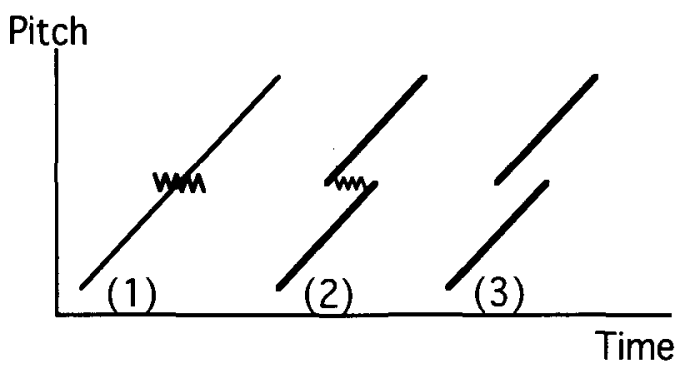

Figure 7. Typical percepts obtained in Experiment 4. 
a convenient tool by which to understand general tendencies in our present experimental paradigm.

Onsets and terminations are not auditory events themselves, and they must be allocated to some events when perceived. Sometimes, they are allocated to auditory events erroneously. That is, an onset and a termination that do not belong to the same physical event can be allocated to the same auditory event when they are close enough in frequency and time to each other. Since the results of Experiments 1, 2, and 3 agreed with each other, we mainly take up the results of Experiment 1 for the present discussion.

The observers in most cases perceived two crossing components, rather than two bouncing components, when they listened to Pattern 1 or 6 . Although this tendency may seem natural at first, it contradicts the widely accepted knowledge that the principle of frequency proximity is dominant, favoring bouncing percepts, in the perception of two crossing frequency glides at identical speeds. According to the event construction model, the onset and the termination of the shorter glide are coupled with each other perceptually because they are close to each other in time and (logarithmic) frequency. Probably, this process is facilitated by the almost continuous distribution of sound energy (or peripheral excitation) that connects the onset and the termination (Figure 8A). Then, the percept of the shorter tone appears as an auditory event. The onset and the termination of the longer glide are left without being interpreted, and they have sufficient sound energy (or peripheral excitation) in between. Thus, the long tone is perceived as a single auditory event. It may seem desirable to define the proximity between an onset and a termination quantitatively in order to examine our hypothesis more systematically, but at this stage of research, we first would like to show that this idea works qualitatively in our present experimental paradigm.

Now we try to apply this model to the gap transfer illusion, which appeared in Patterns 3 and 8. In Pattern 3, the onset of the continuously descending glide and the termination of the first half of the ascending glide are very close to each other in time and (logarithmic) frequency (Figure 8B), and this makes them recouple perceptually, resulting in the percept of a short tone. There is enough sound energy (or peripheral excitation) to connect them without interruption, and this probably facilitates the process. In this case, the auditory system must jump from the descending to the ascending glide somewhere, in order to connect the onset and the termination perceptually. That is, a jump in frequency is required somewhere in the distribution of the presented sound energy (in time and frequency). The jump can be very small. If it occurs just before the termination, for example, it can be as small as half a critical bandwidth. Somewhat larger jumps would be necessary, however, for Patterns $3^{\prime}$ and $8^{\prime}$ in Experiment 3 . The onset of the second half of the ascending glide and the termination of the continuously descending glide are recoupled in the same manner. The first onset and the last termination of the long ascending glide are left without being interpreted perceptually, and they are coupled with each other to form the long ascending tone as an auditory event. In the present model, this long tone is continuous because (1) the termination and the onset around the temporal gap have been interpreted already and (2) the silent period in the long glide has been obscured by the presence of the short glide. The first point cannot be derived easily from the previous research on auditory induction, but this kind of idea is necessary in the present situation, where the gap does not disappear from the whole perceived pattern. The same argument holds for Pattern 8 , where the frequency relationships are reversed.

The auditory system constructs a long ascending or descending tone and two successive short tones for Patterns 3 and 8 . The two short tones are often paired in the same auditory stream because the involved parts in the stimulus pattern are close to each other in time and frequency. If the same auditory stream includes these two tones, it must have a middle part preceded by a termination and succeeded by an onset. The middle part in this context must be a silence, as far as this single auditory stream is concerned. This means that we perceive a temporal gap here.

The perception of Patterns 5 and 10 in Experiments 1 and 2 is suggestive. Both patterns had a temporal gap of about $38 \mathrm{msec}$ at the crossing point. This was a result of the acoustic beats between the two crossing glides. Although the gap did not belong to either glide, the observers tended to report or judge that the shorter perceptual component was far more discontinuous than the longer one. We may assume that weak perceptual clues of a termination and an onset are available at the crossing point in each of these patterns and that the proximity principle works in the same way as in Patterns 3 and 8. The onset of the shorter glide and the weak clue of a termination at the crossing point can be coupled with each other, resulting in the percept of a short tone (Figure $8 \mathrm{C}$ ). The weak clue of an onset - that is, the increase in sound intensity - at the crossing point and the termination of the short glide can be coupled with each other, resulting in the percept of another short tone. Once the clues of a termination and an onset at the crossing point are interpreted, the auditory system basically need not interpret them again, which means that the long ascending component is perceived as continuous. If the physical gap is too long, however, it might be difficult for the auditory system to neglect the clear silent period.

The event construction model thus explains (1) why crossing percepts dominated in Patterns 1 and 6, (2) why the gap transfer illusion took place in Patterns 3 and 8 , and (3) why the temporal gap or dip was not perceived in the longer component as clearly as in the shorter component in Patterns 5 and 10.

The present experimental results showed that onsets and terminations could behave as if they were independent subevents. However, they seem to comply with certain rules regarding their order. For example, a termina- 
(A)

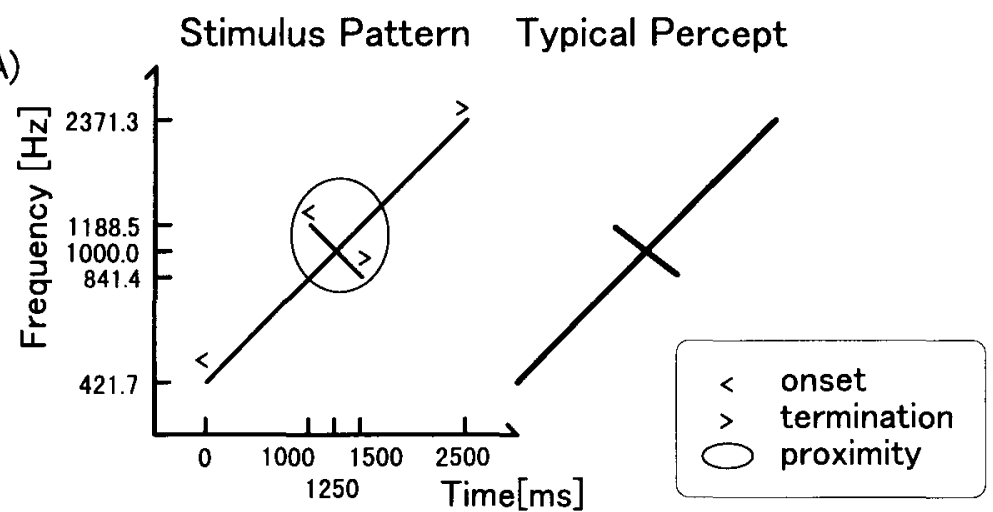

(B)

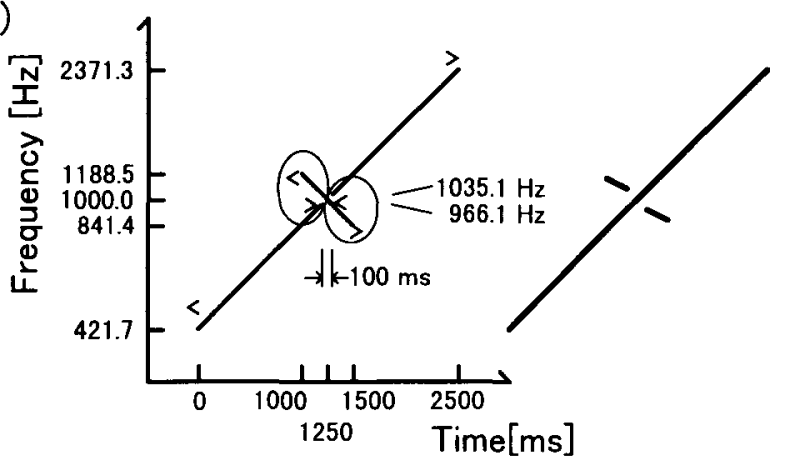

(C)

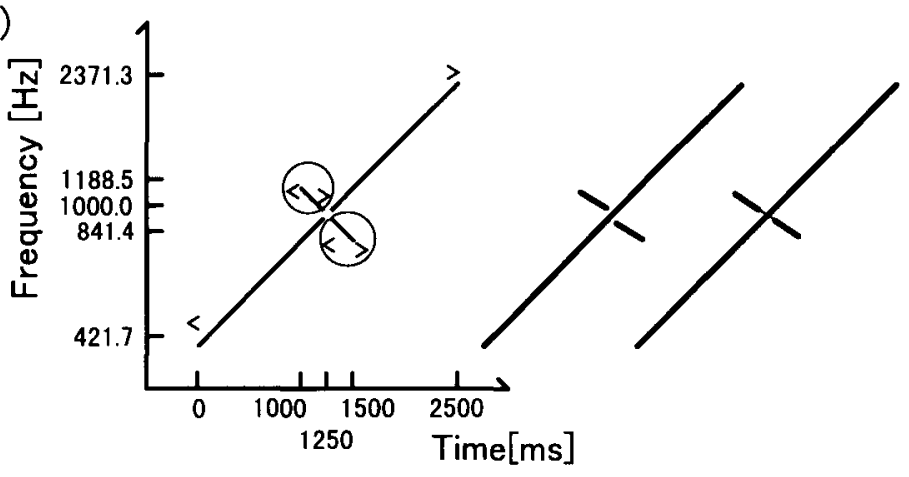

Figure 8. Graphic illustrations of the event construction model. The horizontal axis represents time, whereas the vertical axis represents (logarithmic) frequency or pitch. When an onset $(<)$ and a termination $(>)$ are sufficiently close to each other, they can be coupled as indicated by the circles surrounding them, even when they do not belong to the same physical glide. Thus, an auditory event is constructed.

tion and an onset cannot be connected to each other in this order to form an auditory event, even if they are very close to each other. Kato and Tsuzaki (1998) found that the time interval between an onset clue and a termination clue was more accurately perceived when these clues were available in this order than when the order was reversed. They also found that it was extremely difficult to perceive a time interval between two termination clues. Their results support our fundamental idea that our auditory system utilizes termination clues to construct auditory events by connecting them with preceding onset clues. This idea was proved in a simple paradigm in the present Experiment 4.
The illusory continuity of the long component in Patterns 3 and 8 could be viewed also from another side that is, it seems to reflect the same mechanism as that in the continuity illusion (Warren, 1982, 1999). Probably, the temporal gap in the long glide was filled perceptually with a portion of the short glide. The auditory system could utilize the middle portion of the short glide, which was not necessary to construct the percepts of the two successive short tones. If so, this portion should be in the same frequency region as those of the neighboring parts of the long glide. This kind of frequency proximity indeed seems necessary to generate the perceptual continuity. 
The principle of proximity played an important role in the above discussion, and it seems important to speculate how this principle could appear in the evolutionary process.

\section{REFERENCES}

Bregman, A. S. (1990). Auditory scene analysis: The perceptual organization of sound. Cambridge, MA: MIT Press.

Bregman, A. S. (1993). Auditory scene analysis: Hearing in complex environments. In S. McAdams \& E. Bigand (Eds.), Thinking in sound: The cognitive psychology of human audition (pp. 10-36). Oxford: Oxford University Press.

Buser, P., \& Imbert, M. (1992). Audition. Cambridge, MA: MIT Press.

ELFNER. L. F. (1969). Continuity in alternately sounded tone and noise signals in a free field. Journal of the Acoustical Society of America, 46, 914-917.

HALPERN, L. (1977). The effect of harmonic ratio relationships on auditory stream segregation. Unpublished research report, McGill University, Psychology Department.

HANDEL, S. (1989). Listening: An introduction to the perception of auditory events. Cambridge, MA: MIT Press.

Ka'T, H., \& TsUzakI, M. (1998). Evidence for functional differences between rise and fall markers in discrimination of auditory filled durations. Journal of the Acoustical Society of Japan, 19, 73-76.

McPherson, L. M. P., Ciocca, V., \& Bregman, A. S. (1994). Organization in audition by similarity in rate of change: Evidence from tracking individual frequency glides in mixtures. Perception \& Psychophysics, 55, 269-278.

Miller, G. A., \& LiCKLider, J. C. R. (1950). Intelligibility of interrupted speech. Journal of the Acoustical Society of America, 22, 167 173.

NaKajima, Y., \& SaSaki, T. (1993). Perceptual transfer of onsets and offsets between crossing glide tone components [in Japanese, abstract in English] (Tech. Rep. No. SP92-146, pp. 73-80). Tokyo: Institute of Electronics, Information and Communication Engineers.
NAKAJIMA, Y., \& SASAKI, T. (1996). A simple grammar of auditory stream formation [Abstract]. Journal of the Acoustical Society of America, 100(4, Pt. 2), 2681.

SASAKI, T., \& NAKAJIMA, Y. (1996). An illusory reconstruction of auditory elements [Abstract]. Journal of the Acoustical Society of America, 100(4, Pt. 2), 2751.

Tanaka, S., Nakajima, Y., \& SaSaki, T. (1994). On the mechanism of the gap transfer illusion [in Japanese, abstract in English]. Report of the Acoustical Society of Japan, H-94-72, 1-6.

Thurlow, W. (1957). An auditory figure-ground effect. American Journal of Psychology, 70, 653-654.

Tougas, Y., \& Bregman, A. S. (1985). The crossing of auditory streams. Journal of Experimental Psychology: Human Perception \& Performance, 11, 788-798.

Tsumura, T., Nakajima, Y., \& Teranishi, R. (1991). The temporal processes of auditory excitation produced by frequency modulated tones. Journal of the Acoustical Society of Japan, 12, 43-46.

WARRFN, R. M. (1982). Auditory perception: A new synthesis. New York: Pergamon.

WARREN, R. M. (1999). Auditory perception: A new analysis and synthesis. Cambridge: Cambridge University Press.

Warren, R. M., Bashford, J. A., JR., Healy, E. W. \& Brubaker, B. S. (1994). Auditory induction: Reciprocal changes in alternating sounds. Perception \& Psychophvsics, 55, 313-322.

ZWICKer, E., \& FASTL, H. (1999). Psychoacoustics: Facts and models. Berlin: Springer-Verlag.

\section{NOTE}

1. Related demonstrations are available on the first author's Web site (http://www.kyushu-id.ac.jp/ ynhome/index.html).

(Manuscript received February 18, 1998; revision accepted for publication November 4,1999 .) 